\title{
The Impact of Antimalarial Use on the Emergence and Transmission of Plasmodium falciparum Resistance: A Scoping Review of Mathematical Models
}

\author{
Aleisha R. Brock ${ }^{1, *}$, Carole A. Gibbs ${ }^{2}$ (D), Joshua V. Ross ${ }^{3}$ and Adrian Esterman ${ }^{4,5}$ \\ 1 School of Nursing and Midwifery, Division of Health Sciences, University of South Australia, \\ Adelaide, SA 5000, Australia \\ 2 Library, University of South Australia, Adelaide, SA 5000, Australia; carole.gibbs@unisa.edu.au \\ 3 School of Mathematical Sciences, The University of Adelaide, Adelaide, SA 5005, Australia; \\ joshua.ross@adelaide.edu.au \\ 4 Sansom Institute for Research Health, University of South Australia, Adelaide, SA 5000, Australia; \\ adrian.esterman@unisa.edu.au \\ 5 Australian Institute of Tropical Health and Medicine, James Cook University, \\ Smithfield, QLD 4878, Australia \\ * Correspondence: aleisha.brock@unisa.edu.au; Tel.: +61-8-8302-3958
}

Received: 8 September 2017; Accepted: 6 October 2017; Published: 15 October 2017

\begin{abstract}
The emergence and transmission of resistance to antimalarial treatments continue to hamper malaria elimination efforts. A scoping review was undertaken regarding the impact of antimalarial treatment in the human population on the emergence and transmission of Plasmodium falciparum resistance, to (i) describe the use of mathematical models used to explore this relationship; (ii) discuss model findings; and (iii) identify factors influencing the emergence and transmission of resistance. Search strategies were developed and deployed in six major databases. Thirty-seven articles met the eligibility criteria and were included in the review: nine articles modeled the emergence of resistance, 19 modeled the transmission of resistance, and nine modeled both the emergence and transmission. The proportion of antimalarial use within the population and the presence of residual drug concentrations were identified to be the main predictors of the emergence and transmission of resistance. Influencing factors pertaining to the human, parasite and mosquito populations are discussed. To ensure the prolonged therapeutic usefulness of antimalarial treatments, the effect of antimalarial drug use on the emergence and transmission of resistance must be understood, and mathematical models are a useful tool for exploring these dynamics.
\end{abstract}

Keywords: scoping review; malaria; Plasmodium falciparum; mathematical model; antimalarial resistance; antimalarial treatment; subtherapeutic; drug quality

\section{Introduction}

Malaria is considered to be one of the world's most significant infectious diseases in terms of morbidity and mortality, and is endemic in 91 countries. In 2015, there were approximately 212 million cases of symptomatic malaria worldwide, resulting in 429,000 deaths. Prevalent in parts of Africa, Plasmodium falciparum malaria accounts for $90 \%$ of the global cases and $92 \%$ of global deaths, the majority of which occur in children under five years of age [1]. It is caused by an infection of the red blood cells with plasmodia parasites, with the disease lifecycle occurring in two phases: one within the human host, and one within the female Anopheles species mosquito vector.

The emergence and transmission of resistance to antimalarial treatment continues to hamper malaria elimination efforts. Widespread resistance to antimalarials occurs in two phases: first, 
resistance must emerge within the plasmodia population in order to confer a survival advantage in the presence of drugs; and secondly, resistance must be transmitted through both the human and mosquito populations, which then becomes widespread [2], resulting in treatment failure or death.

The emergence of antimalarial resistance is caused by a random genetic event within plasmodia, whereby a point mutation, or a set of mutations, occurs that may or may not give rise to resistance [3], irrespective of antimalarial treatment [4]. Mutations affording resistance to antimalarial drug(s) can require one or more mutations, which can be linked or unlinked [5]. Intermediate or increasing levels of resistance have been observed through the acquisition of multiple mutations, as with sulfadoxine-pyrimethamine [6] and combination therapy resistance [5].

The survival of resistant plasmodia parasites is determined by natural selection and drug selection. Natural selection refers to the elimination of mutant (including resistant) parasites from direct competition with wild parasites, if the mutation required to produce resistance results in a reduction in fitness compared to competing strains [7]. Drug selection refers to the ability of the parasites to survive in the presence of antimalarial drug, where drug-sensitive parasites will be eliminated, and parasites with resistant-conferring mutations will dominate the infection (competitive release) [6].

Once a resistant strain has emerged and been selected through natural and drug selection, the transmission of resistant plasmodia is very hard to prevent and can have catastrophic effects on the community. In the initial stages of resistance, treatment with the ineffective antimalarial drug continues within the community, as resistance has yet to be detected. This continued ineffective treatment may result in prolonged parasite clearance or treatment failure. Gametocytes with the advantageous mutation(s) will be transmitted to a mosquito during a blood meal [6]. For transmission of the resistant sporozoite plasmodia from the female Anopheles mosquito vector to another human host, the mutant parasite must outcompete other parasite strains in the bottleneck that occurs in the mosquito midgut [8] and survive chromosomal crossover and recombination with the resistant-conferring mutations remaining intact. Chromosomal crossover and recombination can either aid or prevent the continued survival of resistant mutants and occur in the Anopheles mosquito intestinal wall [9]. Chromosomal crossing occurs between homologous chromosomes, in which slices of chromosome are exchanged. Genetic recombination is the process by which genetic material is shared between unlinked and linked nuclear genes. The ability to exchange genetic material aids in the survival of plasmodia by increasing population variability, along with the sharing of any resistant gene(s) required for survival in the presence of antimalarials [10]. However, during this time, the mutated genes conferring resistance may be spliced, and resistance lost. The advantage of antimalarial resistance to a parasite is only apparent under drug selection pressure, where drug sensitive parasites are cleared allowing resistant parasites to dominate the infection [6]. Eventually the resistant parasite population will become established within the infected human and mosquito populations.

Mathematical models have been used to explore the dynamics of malaria for over 100 years [11], and have been used to propose strategies well in advance of comprehensive data becoming available [12].

\section{Study Objective}

The objectives of this scoping review are to: (i) to gather and appraise all available literature that feature mathematical models of the emergence and/or transmission of resistance in P. falciparum that take into account antimalarial use within the human population; (ii) compare and contrast the predicted impact of antimalarial use on the emergence and transmission of resistance, as identified by the models; and (iii) identify key factors that influence this relationship.

\section{Review Methodology}

A scoping review was carried out using a systematic process defined by Arksey and O'Malley (2005) [13], and further enhanced by Levac and colleagues (2010) [14] and Colquhoun and colleagues 
(2014) [15]. The six-step framework proposed by Arksey and O'Malley has been followed, which ensures rigor and transparency, with the sixth step omitted for the purposes of this review [13].

\subsection{Step One: Identifying the Research Question}

In the first stage of the review, three research questions were developed:

1. How have mathematical modeling methods been used to assess the impact of antimalarial treatment in the human population on the emergence and transmission of $P$. falciparum resistance?

2. What risk factors have been identified by mathematical models, of the use of antimalarial treatment in the human population on the emergence and transmission of P. falciparum resistance?

3. What other factors have been identified to influence this relationship, through the use of mathematical modeling?

\subsection{Step Two: Identifying Relevant Studies}

A comprehensive search of the literature was carried out for work published until the end of August 2016, under the guidance of author CAG. The following electronic databases were searched (in order): Medline, Embase, CINAHL, Web of Science, Cochrane Library and Scopus; and grey literature searches (Google Scholar and Open Grey). The MeSH headings and key search terms, along with the combinations used, are provided in Supplementary Materials Tables S1-S6. During full-text screening, the reference lists of relevant articles were also reviewed. All records were exported and managed within Covidence [16].

\subsection{Step Three: Study Selection}

The articles all proceeded through (i) title and abstract screening; and (ii) full-text screening. The inclusion and exclusion criteria are defined below.

\subsubsection{Inclusion Criteria}

- Article must be written in English.

- Publishing date until the end of August 2016.

- The analysis section must contain a mathematical modeling-based approach.

- Results must be provided.

- The human malaria species P. falciparum must be modeled.

- The human host must be studied in the model, with outputs relevant to the human population provided. Other populations, such as the P. falciparum parasite or female Anopheles mosquito vector may also be included.

- The model must explore the effect of an antimalarial agent on the emergence and/or transmission (spread) of antimalarial resistance in P. falciparum.

- Full text must be available.

\subsubsection{Exclusion Criteria}

- Mathematical model not defined in the article.

- Human malaria species: P. vivax, P. malariae, P. knowlesi.

- Results section does not discuss the dynamics in regards to the human population.

- No full text available (e.g., conference abstract, embargoed).

\subsection{Stage Four: Charting the Data}

A data collection form was developed to ensure consistency in data collection from the articles. The data were charted (entered into tables) by summarizing each article reviewed by: author, study aim/objective, model design, key findings, recommendations, and our comments/critique. 
The comments/critique focused on (i) the transparency and reproducibility of the model, given the information provided in the article-for example, are the equations and parameter lists provided? and (ii) the model analysis procedures utilized-for example, whether the findings were validated or whether the model behavior was explored through a sensitivity analysis.

\subsection{Stage Five: Collating, Summarising, and Reporting the Results}

In the final stage of the review, the articles were then categorized as modeling the (i) emergence of antimalarial resistance; (ii) transmission of antimalarial resistance; or (iii) emergence and transmission of antimalarial resistance. The impact of antimalarial use and each category was then assessed and discussed (drug selection pressure), as well as other influencing factors that were taken into account in the model (P. falciparum, host immunity, transmission intensity, and vector control).

\section{Results}

\subsection{Study Selection}

Our search identified 2803 potential articles for the scoping review after duplications were removed, of which 37 articles met the inclusion criteria. Validation of article selection was carried out during the title and abstract screening by author AE. Both authors (ARB and AE) had an agreement rate of $97.8 \%$. Of the articles for which there was a conflict, a Yes/No conflict were found in $66.7 \%$ of the cases and Maybe/No conflict in the remaining 33.3\%. The authors revisited these articles and assigned them accordingly. Full text screening and analysis of results were carried out by author ARB and validated by author JVR. An overview of the study selection process is provided in Figure 1.

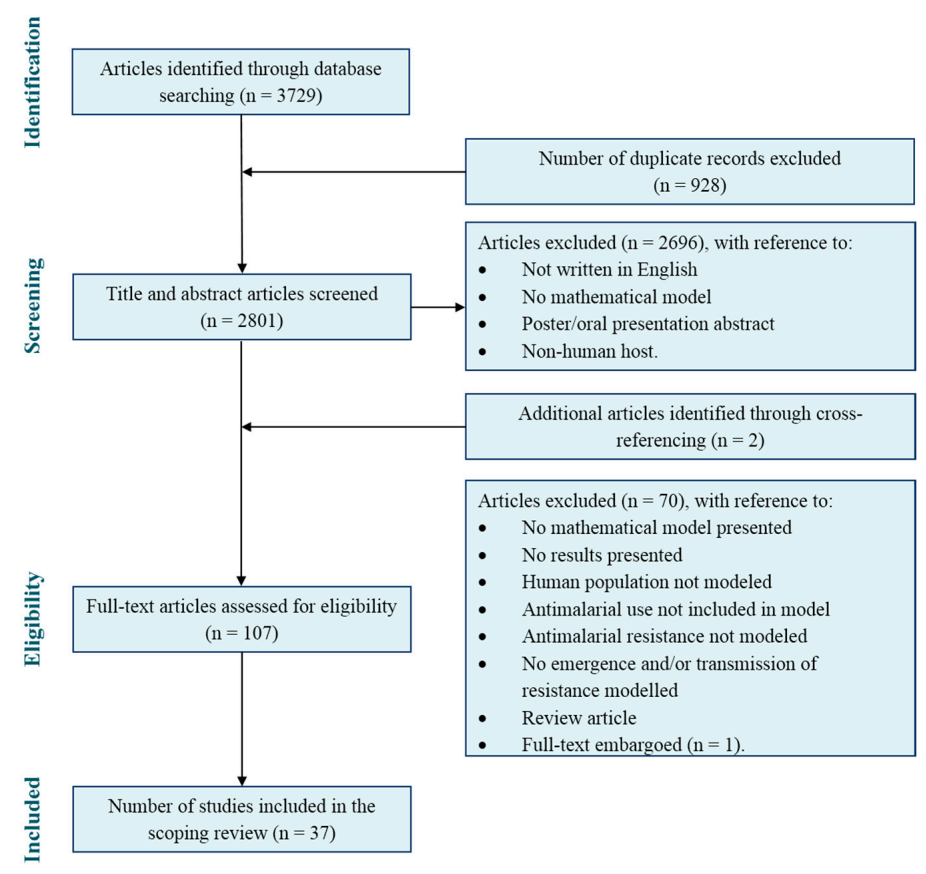

Figure 1. PRISMA flow diagram of the scoping review search and article selection.

\subsection{Mathematical Modelling Methods}

\subsubsection{Model Descriptions}

Of the 37 articles reviewed, nine modeled the emergence of resistance [8,17-24], 19 modeled the transmission of resistance [6,25-42] and nine modeled both [43-51]. A summary of model elements and features is provided in Table 1. 
Table 1. A summary of the model features.

\begin{tabular}{|c|c|c|c|}
\hline \multirow{2}{*}{ Model Feature } & \multicolumn{3}{|c|}{ Frequency [Reference(s)] } \\
\hline & Emergence (Nine Articles) & Transmission (19 Articles) & Both (Nine Articles) \\
\hline \multicolumn{4}{|l|}{ Model Description } \\
\hline \multicolumn{4}{|l|}{ Model type: } \\
\hline Deterministic & $6[8,18-20,23,24]$ & $13[6,25-28,31,33,35,38-42]$ & $6[43-45,48,49,51]$ \\
\hline Stochastic & $3[17,21,22]$ & $5[29,30,32,34,37]$ & $1[46]$ \\
\hline Both & & $1[36]$ & $2[47,50]$ \\
\hline \multicolumn{4}{|l|}{ Scope of model: } \\
\hline Applied & $2[17,23]$ & $4[35,36,39,42]$ & $3[43,49,51]$ \\
\hline Theoretical & $7[8,18-22,24]$ & $15[6,25-34,37,38,40,41]$ & $6[44-48,50]$ \\
\hline \multicolumn{4}{|l|}{ Populations modeled: } \\
\hline Human & & $8[6,30,31,35-37,39,41]$ & $3[48-50]$ \\
\hline Human \& mosquito & $2[17,18]$ & $6[26-28,33,34,40]$ & $4[44,46,47,51]$ \\
\hline Human \& plasmodia & $5[8,19,20,23,24]$ & $3[25,29,42]$ & $1[45]$ \\
\hline Human, mosquito \& plasmodia & $2[21,22]$ & $2[32,38]$ & $1[43]$ \\
\hline \multicolumn{4}{|l|}{ Transparency and reproducibility of model: } \\
\hline Assumptions & $9[8,17-24]$ & $19[6,25-42]$ & 8 [43-51] \\
\hline Equations provided & $7[8,19-24]$ & $17[6,26-28,30-42]$ & $8[43-48,50,51]$ \\
\hline Model flowchart provided & $6[8,17-19,21,22]$ & $18[6,26-29,31-41]$ & $5[43,46,48,49,51]$ \\
\hline Model validation & $2[17,23]$ & $5[26,27,29,35,42]$ & $2[43,45]$ \\
\hline Parameters provided & $8[8,17,19-24]$ & $16[6,26-28,30-32,34-42]$ & $8[43-47,49-51]$ \\
\hline Sensitivity analysis & $2[19,21]$ & $5[26,29,30,36,37]$ & $2[47,50]$ \\
\hline \multicolumn{4}{|l|}{ Antimalarial Resistance } \\
\hline \multicolumn{4}{|l|}{ Monotherapy } \\
\hline Artemisinin & $1[8]$ & $3[35,39,42]$ & $1[49]$ \\
\hline Artesunate & & $3[6,35,36]$ & $1[43]$ \\
\hline Atovaquone & & & $1[49]$ \\
\hline Chloroproguanil-dapsone & & $1[41]$ & \\
\hline Chloroquine & & $1[42]$ & $1[43]$ \\
\hline Lumefantrine & & $1[42]$ & \\
\hline Mefloquine & $1[23]$ & $1[42]$ & \\
\hline Piperaquine & & $5[6,35,36,39,42]$ & \\
\hline Pyrimethamine & $1[17]$ & & \\
\hline Quinine & & & $1[43]$ \\
\hline Not specified & $2[18,22]$ & $3[25,27,34]$ & \\
\hline \multicolumn{4}{|l|}{ Combination therapy } \\
\hline Artemisinin-based combination therapy (ACT) & & $1[39]$ & $1[50]$ \\
\hline Sulfadoxine-pyrimethamine & $1[24]$ & $4[25,29,39,41]$ & $2[43,45]$ \\
\hline Not specified & $2[18,22]$ & $2[25,34]$ & \\
\hline Partner-drug resistance (not specified) & $2[8,24]$ & $3[34,35,37]$ & \\
\hline Resistance type not specified & $2[19,20]$ & $8[26,28,30-33,38,40]$ & $5[44,46-48,51]$ \\
\hline Degree of resistance specified (partial, full) & $3[21,23,24]$ & $5[6,29,39-41]$ & $3[45,46,51]$ \\
\hline \multicolumn{4}{|l|}{ Drug Selection Pressure } \\
\hline Antimalarial treatment: & & & \\
\hline Monotherapies & & & \\
\hline Artemisinin & $1[8]$ & & \\
\hline Artesunate & & $1[36]$ & $2[43,49]$ \\
\hline Chloroproguanil-dapsone & $1[24]$ & $2[6,41]$ & \\
\hline Chloroquine & & $1[42]$ & $1[43]$ \\
\hline Lumefantrine & & $1[42]$ & \\
\hline Mefloquine & $1[23]$ & $1[42]$ & \\
\hline Piperaquine & & $1[42]$ & \\
\hline Pyrimethamine & $1[17]$ & & \\
\hline Quinine & & & $1[43]$ \\
\hline Not specified & $2[18,22]$ & $3[25,27,34]$ & \\
\hline Combination therapies & & & \\
\hline Artemether-lumefantrine & & $1[42]$ & \\
\hline Artemisinin-based combination therapy (ACT) & $1[8]$ & $1[37]$ & $2[49,50]$ \\
\hline Artemisinin-piperaquine & & $1[35]$ & \\
\hline Artemisinin-piperaquine + primaquine & & $2[35,36]$ & 1 [49] \\
\hline Artesunate + piperaquine & & $1[36]$ & \\
\hline Artesunate + mefloquine & & $1[42]$ & \\
\hline Artesunate + chloroquine & & $1[42]$ & \\
\hline Artesunate-lumefantrine & & $1[42]$ & \\
\hline Atovaquone + progunail & & $1[36]$ & 1 [49] \\
\hline Atovaquone + progunail + primaquine & & $1[36]$ & $1[49]$ \\
\hline Chloroproguanil-dapsone + artesunate & $1[24]$ & & \\
\hline Dihydroartemisinin + piperaquine & & $2[39,42]$ & \\
\hline Sulfadoxine-pyrimethamine & $1[24]$ & $3[6,29,41]$ & $2[43,45]$ \\
\hline Sulfadoxine-pyrimethamine + amodiaquine & $1[24]$ & & \\
\hline Sulfadoxine-pyrimethamine + artesunate & $1[24]$ & $1[39]$ & $1[45]$ \\
\hline Chloroproguanil-dapsone + artesunate & & & 1 [45] \\
\hline Not specified & $2[18,22]$ & $2[25,34]$ & \\
\hline
\end{tabular}


Table 1. Cont.

\begin{tabular}{|c|c|c|c|}
\hline \multirow{2}{*}{ Model Feature } & \multicolumn{3}{|c|}{ Frequency [Reference(s)] } \\
\hline & Emergence (Nine Articles) & Transmission (19 Articles) & Both (Nine Articles) \\
\hline \multicolumn{4}{|l|}{ Combination therapies } \\
\hline Artemether-lumefantrine & & $1[42]$ & \\
\hline Artemisinin-based combination therapy (ACT) & $1[8]$ & $1[37]$ & $2[49,50]$ \\
\hline Artemisinin-piperaquine & & $1[35]$ & \\
\hline Artemisinin-piperaquine + primaquine & & $2[35,36]$ & $1[49]$ \\
\hline Artesunate + piperaquine & & $1[36]$ & \\
\hline Artesunate + mefloquine & & $1[42]$ & \\
\hline Artesunate + chloroquine & & $1[42]$ & \\
\hline Artesunate-lumefantrine & & $1[42]$ & \\
\hline Atovaquone + progunail & & $1[36]$ & 1 [49] \\
\hline Atovaquone + progunail + primaquine & & $1[36]$ & $1[49]$ \\
\hline Chloroproguanil-dapsone + artesunate & $1[24]$ & & \\
\hline Dihydroartemisinin + piperaquine & & $2[39,42]$ & \\
\hline Sulfadoxine-pyrimethamine & $1[24]$ & $3[6,29,41]$ & $2[43,45]$ \\
\hline Sulfadoxine-pyrimethamine + amodiaquine & $1[24]$ & & \\
\hline Sulfadoxine-pyrimethamine + artesunate & $1[24]$ & $1[39]$ & $1[45]$ \\
\hline Chloroproguanil-dapsone + artesunate & & & $1[45]$ \\
\hline Not specified & $2[18,22]$ & $2[25,34]$ & \\
\hline Treatment not specified & $3[19-21]$ & $8[26,28,30-33,38,40]$ & $5[44,46-48,51]$ \\
\hline \multicolumn{4}{|l|}{ Antimalarial treatment strategies: } \\
\hline Intermittent-preventive treatment (IPT) & & $3[6,39,41]$ & $1[49]$ \\
\hline Mass drug administration (MDA) & & $2[35,36]$ & 1 [49] \\
\hline Mass screening and treatment (MSAT) & & $1[36]$ & \\
\hline \multicolumn{4}{|l|}{ Antimalarial properties and duration of treatment: } \\
\hline Full/partial treatment duration & & $1[25]$ & \\
\hline Half-life/decay of concentration with time & $2[22,23]$ & $3[6,41,42]$ & $2[43,45]$ \\
\hline High/low dose & $2[22,23]$ & & $1[49]$ \\
\hline Residual levels & $2[8,23]$ & 1 [41] & \\
\hline Levels of drug efficacy & $1[17]$ & & \\
\hline Parasite growth restriction following treatment & $1[22]$ & & \\
\hline Patient compliance & & $1[37]$ & \\
\hline Protection from reinfection & $1[24]$ & & \\
\hline Transmissibility following treatment & $1[22]$ & & \\
\hline \multicolumn{4}{|l|}{ Potential Influencing Factors } \\
\hline \multicolumn{4}{|l|}{ Plasmodium falciparum: } \\
\hline Asexual parasite density & $3[17,22,23]$ & $1[29]$ & \\
\hline Epistasis & & $1[25]$ & \\
\hline Frequency of mutation & & $1[25]$ & \\
\hline Gametocyte parasite density & $3[17,22,24]$ & $1[29]$ & \\
\hline Genetic recombination & $3[18,20,22]$ & $2[25,34]$ & \\
\hline Inbreeding and/or random mating & & $3[25,34,38]$ & \\
\hline Infectivity/transmissibility following treatment & $1[17]$ & $4[28,29,31,34]$ & \\
\hline Parasite fitness & 4 [19-22] & $12[6,25-27,30-34,38,40,41]$ & $6[44,46-48,50,51]$ \\
\hline Multiplicity of infection (MOI) & & $1[32]$ & \\
\hline Mutation rate & $1[22]$ & & \\
\hline Natural selection & $1[22]$ & $3[26,30,32]$ & $1[46]$ \\
\hline \multicolumn{4}{|l|}{ Host immunity: } \\
\hline Acquired/clinical immunity or host age-dependent & $4[8,17,21,23]$ & $13[6,25-28,31,33,35,36,39-42]$ & $3[43,46,49]$ \\
\hline Immune response & $1[19]$ & & $1[47]$ \\
\hline Generalized/strain specific immunity & $1[20]$ & & $1[47]$ \\
\hline Symptomatic and/or asymptomatic infection & $1[22]$ & $7[6,29,31,35,36,39,41]$ & \\
\hline Transmission intensity & $3[8,21,24]$ & $11[6,25,26,31,32,36-39,41]$ & $1[45]$ \\
\hline \multicolumn{4}{|l|}{ Female Anopheles mosquito: } \\
\hline Competition for blood meal & & & $1[43]$ \\
\hline Entomological inoculation rate (EIR) & & $5[31,32,37,41,42]$ & \\
\hline Fitness of mosquitoes to produce offspring & $1[21]$ & & \\
\hline Insecticide resistance & $1[21]$ & & \\
\hline Population size dependent on climatic factors & $2[17,21]$ & $1[26]$ & \\
\hline Sporozoite measure (count/rate) & & $1[34]$ & \\
\hline Transmission blockers: & & & 1 [45] \\
\hline Insecticidal bednets & & $3[35,36,39]$ & $1[49]$ \\
\hline Transmission potential & $1[21]$ & $1[26]$ & \\
\hline Vectorial capacity & & $4[26,31,32,37]$ & \\
\hline
\end{tabular}

The most common form of mathematical modeling was deterministic modeling $(25 / 37,68 \%)$, with stochastic modeling used in nine articles ( $24 \%$ ), and a combination of deterministic and stochastic modeling techniques used in three $(8 \%)$ articles. Deterministic models contain no random probabilistic elements, providing constant model outcomes for a specific set of initial values and parameters; unlike stochastic models, which sample from a probability distribution for each parameter and allow for random variation.

Twelve (32\%) articles modeled the dynamics within the human and mosquito populations, predominantly to take into account transmission. The human population alone was modeled in $11(30 \%)$ articles; interestingly, none of these models explored the dynamics of the emergence of 
resistance. The human and P. falciparum populations were modeled in nine (22\%) articles; and the remaining five $(14 \%)$ articles modeled the human, mosquito and P. falciparum populations.

The majority of the articles used the literature to inform parameter values $(28 / 37,76 \%)$, whereas nine articles used field, clinical or laboratory observations from Tanzania, Uganda, Sudan, Burkino Faso, Cambodia, and Thailand.

The model equations were provided in $86 \%(32 / 37)$ of the articles (whether in text or supplementary), and a list of parameter values (tabulated or in text) were provided in $86 \%(32 / 37)$ of the articles. The model assumptions were outlined in every article reviewed (100\%). An analysis of model behavior in the form of assessing the sensitivity of the model outputs to parameter values (sensitivity analysis) and/or validating the model outputs through a comparison of key outputs to literature, laboratory or clinical data observations (model validation), were presented in $62 \%(23 / 37)$ of articles.

\subsubsection{Antimalarial Resistance}

Antimalarial resistance to 11 monotherapy treatments were modeled (emergence: three treatments; transmission: seven treatments; emergence and transmission: five treatments), with five articles not specifying the type of monotherapy treatment (emergence: two articles [18,22]; transmission: three articles $[25,27,34])$. Resistance to combination therapies were modeled in 13 articles: artemisinin-based combination therapy (ACT) resistance in two articles, sulfadoxine-pyrimethamine (SP) resistance in seven articles, and four articles failed to specify the combination therapy to which resistance was present within the population $[18,22,25,34]$. There were 15 articles that did not specify the antimalarial treatment to which resistance existed. Partner-drug resistance was explored in five articles. Differing levels of resistance (partial, full and/or varying numbers of mutations affording resistance) were modeled in 11 articles.

\subsubsection{Antimalarial Treatment}

Drug selection pressure was modeled through the use of antimalarial treatments, treatment strategies, and antimalarial properties. The type of antimalarial treatments modeled in the articles included nine different antimalarial monotherapy treatments and 16 different antimalarial combination therapies. Antimalarial treatment was not specified in 16 articles, with an additional five articles not specifying the type of monotherapy treatment, and four articles not specifying the type of combination therapy modeled. Multiple treatments (monotherapy and/or combination therapy) were modeled in 16 articles: 11 articles modeled monotherapies and combination therapies [6,8,18,22,24,25,34,36,41,43,49]; two articles modeled more than one monotherapy treatment [42,43]; and six articles modeled more than one combination therapy $[8,24,35,36,39,42]$. Antimalarial treatment strategies were explored in transmission and emergence and transmission models, namely the use of intermittent preventive treatment (IPT) in four articles [6,39,41,49], mass drug administration (MDA) in three articles [35,36,49], and mass screening and treatment (MSAT) in one article [36]. Antimalarial properties, such as the drug half-life, residual drug levels, dosage and treatment compliance, were modeled in 12 articles.

\subsubsection{Potential Influencing Factors}

Other factors included in the mathematical models that have the potential to influence the relationship between resistance and antimalarial use, were P. falciparum specific factors (within-host competition, natural selection, parasite fitness), human immunity, transmission intensity, female Anopheles mosquito factors and transmission blocking mechanisms.

\subsection{Risk Factors for the Emergence of Antimalarial Resistance Identified by Mathematical Models}

Mathematical modeling was identified to explore the dynamics of the emergence of antimalarial resistance, taking into account antimalarial treatment use within the host population in nine articles [8,17-24]. The emergence of resistance was predicted to increase in response to: 
- An increase in the proportion of the population who receive antimalarial treatment (population coverage) [17,18];

- A shorter duration of high-dose active pharmaceutical ingredient (API) [22];

- A lower drug efficacy [22];

- $\quad$ Residual drug concentrations (sub-optimal API) [8,23];

- The use of monotherapies compared to combination therapy [18,24];

- Treatment timing $[19,22]$;

- $\quad$ A higher P. falciparum mutation rate [22];

- A lower metabolic cost of resistance in P. falciparum [22]; and

- A decrease in host natural immunity (corresponding to areas of lower transmission intensity or endemicity) [21,23].

\subsubsection{Drug Selection Pressure}

Antimalarial use was found to be a major contributor to the emergence of resistance $[8,18]$. The blood stage replication of $P$. falciparum parasites was identified to be the most likely lifecycle stage for the emergence of resistance, predicted to be up to five times higher in the presence of antimalarials, especially in areas of low transmission [8]. The exposure of $P$. falciparum parasites to residual drug concentrations (sub-optimal API) was also identified to be an important risk factor for selecting resistance [8,23], especially in areas of high transmission [8].

The emergence of resistance was predicted in response to an increase in the population treatment coverage and the use of higher doses (API). This was observed through the modeled use of pyrimethamine (monotherapy), with the model findings recommending that the administration of high doses should be restricted to less than $25 \%$ of the population [17].

The use of combination therapy was modeled in three articles and is predicted to decrease the emergence of resistance $[18,22,24]$. This decrease assumes that (i) both resistances are initially rare; (ii) genetic recombination between resistant genes occurs; and (iii) there is low treatment coverage. However, if no recombination between resistance genes is permitted within the model, combination therapy was not found to be advantageous [18].

The choice of partner drug was identified to be an important factor in prolonging the therapeutic usefulness of combination therapies and preserving the reduction in the chance of the emergence of resistance. Once resistance to one of the partner drugs emerges, there is a decrease in the ability of the combination therapy to delay the emergence of resistance to the other drug. Additionally, the use of a partner drug that had been used as a monotherapy, or one to which the parasite population had been previously exposed, predicted an increase in the time to emergence of resistance [24]. Further, the addition of a partner drug to an already failing treatment did not significantly increase the useful therapeutic life.

Antimalarial dose was found to impact the predicted time to the emergence of resistance. The modeled use of low-dose mefloquine (monotherapy) predicted a faster emergence of resistance than a high dose. The use of low dose resulted in more parasites at all times and increased the opportunity for the selection of mutant $P$. falciparum parasites [23].

\subsubsection{Plasmodium falciparum}

An increase in P. falciparum mutation rates was identified to decrease the time to the emergence of resistance; however, the mutation rate is thought to be independent of treatment type or use [5]. An increase in the PfEMP1 var gene switching was also identified to increase the time to the emergence of resistance [19]. 


\subsubsection{Host Immunity}

Antimalarial drugs act synergistically with the host immune defenses [6], and when taking this into account, the timing of treatment was found to be critical for the prevention of the emergence of resistant parasites [19]. A within-host model predicted an increase in the likelihood of a mutant parasite developing into a viable drug-resistant population as a result of early treatment before parasite density triggers anti-PfEMP1 (immune) response, and a high antibody threshold with a long lag time between antibody simulation and activity [19]. In contrast, a stochastic model suggested that early treatment predicted a decrease in the probability of emergence [22].

\subsubsection{Transmission Intensity}

The acquisition of host immunity occurs through repeated exposure to malaria. In areas of high transmission intensity (endemicity), a greater proportion of the population is thought to have a higher level of immunity [5]. This increase in host immunity results in more asymptomatic infections, which in turn, results in less antimalarial use within the population, decreasing the drug selection pressure $[4,52]$. The emergence of resistance was predicted to be higher in areas of low transmission than high transmission [8]. In areas with greater levels of host immunity, it was predicted that even when there were low levels of resistance present, the use of low antimalarial dose (API) resulted in more patients being cured [23].

The effect of projected climate change was identified to have an indirect impact on the time to the emergence of resistance, by increasing the transmission intensity, which results in an increase in host immunity (and hence, a decrease in drug selection pressure) [21].

\subsubsection{Vector Control}

The use of insecticides to control mosquito populations in low transmission areas were predicted to decrease the time to the emergence of resistance, when used in conjunction with antimalarial drugs. However, in high transmission settings, these same interventions were predicted to enhance the emergence of resistance within the population [21].

\subsection{Risk Factors for the Transmission of Antimalarial Resistance Identified by Mathematical Models}

Mathematical modeling was identified to explore the dynamics of the transmission of antimalarial resistance taking into account antimalarial treatment use within the host population in 19 articles [6,25-42]. The transmission of resistance was predicted to increase with:

- An increase in the proportion of the population who receive antimalarial treatment (population coverage) $[6,27,35,36,38]$, including intermittent preventive treatment [6] and mass drug administration strategies [35,36];

- The use of monotherapies compared to combination therapy [35-37,42];

- An increase in presumptive antimalarial use [37];

- A longer drug half-life [6,41];

- Residual drug concentrations [29,37];

- An increase in the infectious periods of hosts [28];

- A decrease in the recovery rate of nonimmune humans infected by resistant parasites [27];

- Increasing level of resistance (i.e., the number of mutated alleles conferring resistance, partial- or full-resistance) $[29,40]$;

- A decrease of within-host competition between drug-resistant and drug-sensitive P. falciparum parasites [32];

- A decrease in the fitness cost associated with resistance [25,27,30,32];

- An increase in the lifespan of resistant-infected mosquitoes [27];

- An increase in mosquito diffusion [27]; 
- An increase in the number of sporozoites injected from an infected female Anopheles mosquito to susceptible human per blood meal [34]; and

- $\quad$ A decrease in the use of transmission blockers (e.g., bednets) [36].

\subsubsection{Drug Selection Pressure}

An increase in the proportion of antimalarial use was identified as the most important predictor of the transmission of resistance [34]. The existence of a treatment threshold was explored, which was determined by the rates of infection and the infectious periods of resistant and sensitive infections in treated and untreated populations, and findings suggest that resistance will spread and become fixed within the population once the treatment coverage is greater than the threshold [33]. The occurrence of treatment failure (the inability to clear parasites or resolve clinical symptoms of malaria, despite antimalarial treatment administration [4]) was identified as a predictor of the transmission of resistance [37]. Late clinical failure or late parasitological failure as a result of resistance was found to increase the time an individual remained infectious by a few months, whereas low parasite clearance was only found to increase this by a few days [39]. The use of antimalarial treatment was identified to reduce the average duration of sensitive and partially resistant infections [41].

The continued use of monotherapies for which resistance was already established within the population, resulted in an increase in the proportion of resistant infections and the prevalence of malaria infections [36]. The use of ACTs resulted in a long-term reduction in the overall incidence of malaria [35] and the transmission of resistance [37]. The pharmacokinetic properties of partner drugs in ACTs were identified to be the most important determinant of treatment outcome, followed by the rate of parasite growth [42]. For example, an increase in artemisinin $\mathrm{IC}_{50}$ (the concentration required to decrease parasitaemia by half) resulted in an increase in resistance, either due to reduced protection to the partner drug or an increase in treatment failure [42].

Partner drug resistance was found to reduce the effectiveness of combination therapies. The use of ACTs (treatment or MDA) in an area with high levels of artemisinin resistance predicted a decrease in treatment effectiveness [35]. Should the use of good quality ACT continue at a high level within the population, even in the presence of artemisinin or partner drug resistance, the model predicted that malaria elimination was possible (note: artemisinin resistance increased to $82-100 \%$ of infections prior to malaria elimination) [36].

The use of intermittent prophylaxis administration (IPT) was identified to increase the transmission of resistance, more so in infants than adults (i.e., pregnant women) [6]. Mass drug administrations (MDA) were also identified to increase the transmission of resistance [35] and were not identified to be an effective treatment strategy (alone) to eradicate malaria [36].

The transmission of resistance was predicted to increase with an increase in half-life [6,41]. Similarly, the transmission of resistance was found to increase with mismatched half-lives of partner drugs used in combination therapies [37].

The proportion of human population with residual drug levels was identified to be a driver of the transmission of resistance in high transmission areas, providing the resistant parasites with a survival advantage [37]. In the presence of residual concentrations, highly mutated parasites conferring sulfadoxine-pyrimethamine resistance were predicted to recrudesce earlier. In contrast, when there were fewer mutations, exposure to residual sulfadoxine-pyrimethamine concentrations resulted in sub-patent parasitemia levels $(<10$ parasites $/ \mathrm{mL})$ in the weeks following treatment, which allowed the host's specific immune response to further control parasitaemia levels, delaying the recrudescence of clinical symptoms [29].

The transmission of resistance was dependent on the transmission rates of sensitive and resistant infections (the ratio of the infectious periods of treated and untreated infections). If the host becomes immediately non-infectious (cannot transmit gametocytes) following treatment, an increase in population treatment coverage results in a decrease in the number of sensitive infections, and an increase in the transmission of resistance. If the host remains infectious post-treatment (still able to 
transmit gametocytes), the transmission of resistance is dependent on the basic reproduction rate $\left(\mathrm{R}_{0}\right)$ of sensitive and resistant infections [28].

\subsubsection{Plasmodium falciparum}

The speed of transmission is predicted to increase as the basic reproduction rate $\left(R_{0}\right)$ of drug-resistant parasites becomes larger than the $\mathrm{R}_{0}$ of drug-sensitive parasites [27]. The biological cost of resistance (fitness cost) was identified to be less important in predicting the transmission of resistance, than the duration of drug-resistant infections [32]. The forces of intra-host selection and parasite fitness were found to work in opposite directions, and change in magnitude as the frequency of resistance increases. When there were low levels of resistance within the population, the net force increased, resulting in an increase in resistance levels. As resistance frequency increased, the net force decreased and eventually become negative. This net force drives the stabilization of resistance (below $100 \%$ ) within the population (when the net force equals zero). Under drug selection pressure, the balance of forces was tipped towards the transmission of resistance, which reached 100\% [30].

The more tolerant the mutant parasite is to the drug, the less the drug will kill [40], resulting in a greater competitive release compared to the drug-sensitive parasites. The degree of resistance is determined by the concentration of antimalarial drug that the parasite can survive, and may be encoded by one or multiple mutations. Increasing levels of resistance, commonly identified by the number of mutations present that confer resistance, were identified to influence the potential for the transmission of resistance [29]. When resistance was encoded by a mutation at one locus, additional mutated loci were predicted to be a disadvantage [25].

In areas of high treatment coverage ( $80 \%$ of clinical infections treated), the ability for resistant parasites to outcompete and dominate the infection decreases with increasing transmission. In areas of low transmission intensity, resistant parasites were predicted to dominate at all fitness costs. However, as transmission intensity increases, there is a decrease in the range for which the drug-resistant parasites dominate. In areas of low treatment coverage ( $20 \%$ of clinical infections treated), coexistence between resistant and sensitive parasites occurs in areas of low transmission intensity (but resistant parasites do not exclude sensitive), and once again, the range of fitnesses for which coexistence is predicted to occur decreases with increasing transmission intensity [32].

As treatment coverage increased, the proportion of infections with an intermediate level of resistance was predicted to increase [40]. The investigation of a range of dihydrofolate reductase (DHFR) mutations, associated with sulfadoxine-pyrimethamine resistance, predicted the highest transmission potential in moderately-resistant parasites due to a delay in recrudescence and clinical symptoms. However, when exposed to residual concentrations, it was identified that highly-mutated parasites recrudesced earlier [29].

\subsubsection{Host Immunity}

Host immunity was identified to be a predictor of the transmission of resistance $[6,27,28]$. Partial immunity was predicted to increase the $\mathrm{R}_{0}$ of sensitive parasites [28] and reduce the disease incidence (due to more asymptomatic infections) [28]; however, transmission from asymptomatic infections still continued [28]. An increase in the transmission of resistance was also predicted as a result of the increase in the recovery time of non-immune resistant-infected humans [27].

\subsubsection{Transmission Intensity}

The degree of transmission intensity (endemicity) in an area was not identified to be predictive of the transmission of resistance [27,28]; however, it is suggested to indirectly influence it through its influence on the acquisition of host immunity and corresponding antimalarial use [26,31]. In areas of low transmission intensity, an increase in drug selection pressure is observed, due to more symptomatic infections requiring treatment (greater population treatment coverage or drug selection pressure). This resulting increase in treatment is suggested to drive the observed difference between low and 
high transmission intensity areas. As a result of the drug selection pressure observed in areas of low transmission intensity, drug-resistant parasites were predicted to spread and eventually dominate the drug-sensitive parasites [32], and hence, the transmission of resistance was predicted to be greater compared to areas of high transmission intensity [37]. Low transmission intensity areas or areas of unstable transmission were also predicted to coincide with outbreaks of resistance [31].

Other models identified that areas of high transmission intensity were a reservoir for sensitive parasites [31] due to the increase in asymptomatic infections, and corresponding to less drug pressure [26]. This finding held true when the $R_{0}$ of resistant parasites was greater than the $R_{0}$ of sensitive parasites, as the population-wide immunity helped to prevent the transmission of resistance [31]. Another model predicted that partially-resistant parasites were more prevalent in low transmission intensity areas, and fully-resistant parasites in high transmission intensity areas [6].

When areas of intermediate transmission intensity were added to the models, a non-monotonic pattern was observed. In this situation, drug-resistant parasites were favored in areas of low and high transmission intensity, and drug-sensitive parasites in areas of intermediate transmission intensity. The model exhibited a second threshold for the re-establishment of resistance, determined by the cost of resistance, drug coverage, and the fitness of resistant parasites under treatment [26].

Asymptomatic infections, in combination with high transmission intensity, allowed for sulfadoxine-pyrimethamine-resistant parasites (three DHFR mutations and less in DHPS conferring sulfadoxine-pyrimethamine resistance) to expand largely unnoticed [29]. This finding was supported in two other models, which predicted that resistant parasites were more likely to be transmitted in high transmission areas [6,34].

\subsubsection{Vector Control}

The mosquito factors for which the transmission of resistance was dependent on were: the death rate of mosquitoes infected with resistant parasites [27], the diffusion of mosquitoes coefficient [27], the number of sporozoites per mosquito [34], and the use of transmission blockers, such as insecticide-treated bednets (ITNs) [36]. The use of ITNs, in combination with good quality ACT with high treatment coverage, reduced transmission by $30 \%$, and reduced the time to eradication of malaria by $50 \%$ [36].

\subsection{Risk Factors for the Emergence and Transmission of Antimalarial Resistance Identified by Mathematical Models}

Mathematical modeling was identified to explore the dynamics of the emergence and transmission of antimalarial resistance taking into account antimalarial treatment use within the host population in nine articles [43-51]. The emergence and transmission of resistance is predicted to increase with:

- An increase in the proportion of the population who receive antimalarial treatment (population coverage) $[43,50,51]$;

- $\quad$ Longer drug half-lives [43];

- Residual drug concentrations [45];

- An increased rate of parasite mutation [43];

- An increased relative fitness of resistant P. falciparum parasites compared to their drug-sensitive counterparts [44];

- A decrease in transmission intensity [47]; and

- The decreased use of transmission blockers (i.e., bednets) [49].

\subsubsection{Drug Selection Pressure}

An increase in the use of antimalarial treatments was predicted to increase the establishment of resistance within the population [50]. The predicted time to the emergence and establishment of resistance was predicted to halve in response to double the amount of antimalarial use [50]. 
Model findings suggested that the emergence of partial resistance was primarily determined by the proportion of infections treated $[45,50]$ and the proportion with residual drug levels within the population [45]. The emergence of full resistance was primarily determined by the proportion of infections treated [45]. It was shown that resistance to the antimalarial treatment first given will emerge and transmit, and will only decrease if treatment changes [44].

Treatment influences the transmission of resistance, with a trade-off between slowing resistance and curbing disease incidence [47]. The rate of transmission of resistance was predicted to increase in response to an increase in antimalarial use [50]; however, treatment coverage primarily affected disease prevalence [47] and treatment efficacy primarily affected the transmission of resistance [47]. The transmission rate of partially-resistant parasites was predicted to be slower than fully-resistant parasites; however, partially-resistant parasites were more likely to be initially present at higher frequencies [45]. The classical requirement of having $R_{0}<1$ to prevent the transmission of malaria was not upheld when multiple levels of resistance were present within the population, suggesting that an increase in treatment has a limited benefit, especially in high transmission settings [51].

The use of combination therapy was predicted to decrease the emergence and transmission of resistance [44,49]. However, the use of a combination therapy to which resistance to one of the partner drugs was already established, resulted in an increased time for the establishment and transmission of resistance within the population [49]. MDA efficacy was predicted to decrease with an increase in the population treatment coverage [49].

\subsubsection{Plasmodium falciparum}

There is evidence of a threshold for determining which strain will dominate, which is dependent on the fitness of the resistant strain, the infection period and treatment rate [44]. The continued treatment with an ineffective antimalarial to which resistance has emerged is predicted to increase the proportion of infections in each class of resistance present [51].

\subsubsection{Transmission Intensity}

The proportion of resistant infections was predicted to be lower in areas of high transmission intensity than low transmission intensity [47].

\subsubsection{Vector Control}

The modeled use of transmission blockers provided mixed results. A decrease in the emergence and transmission of resistance was predicted in response to an increase in ITN use in one model [49]; however, the use of transmission blockers (e.g., bednets and vaccination programs) was not predictive of the emergence of resistance in another [45]. It was further suggested that the use of transmission blockers may be indirectly related to the emergence of resistance through the corresponding reduction of antimalarial use associated with transmission blockers [45].

\section{Discussion}

The scoping review identified 37 articles containing mathematical models exploring the dynamics of the emergence and/or transmission of antimalarial resistance, taking into account the use of antimalarial treatment in the host (human) population. The model findings identified that the drug selection pressure exerted by the use of antimalarial treatments plays an important role in the emergence and transmission of resistance. Further, commonly-identified drivers were the proportion of antimalarial use within the population and residual drug levels (sub-optimal/sub-therapeutic API). Factors that contribute to a greater drug selection pressure on the system are of importance for the assessment of their impact, and for determination of areas of intervention. 


\subsection{Mathematical Models}

A range of mathematical models was used to explore the dynamics of the emergence and/or transmission of antimalarial resistance. Deterministic modeling was the most common type $(68 \%)$, with an additional three articles $(8 \%)$ using a combination of deterministic and stochastic modeling techniques.

An analysis of model behavior, sensitivity of parameters, and validation of model outputs were presented in over half of the manuscripts, which are vital for understanding the stability of the model and reliability of the findings. The model assumptions were provided in all the articles reviewed. The transparency and reproducibility of the mathematical models allow for rigorous review of the modeling methods utilized, underlying assumptions and a more in-depth understanding of the inputs that drive the model dynamics and their limitations. We observed a general increase in the transparency and reproducibility of models over time, with the inclusion of model equations, parameter values, and model flow diagrams within the manuscripts (or Supplementary Materials).

There is a tradeoff between the complexity of a mathematical model and the interpretation of results. Model complexity was added through the populations modeled and the factors explored. The nature of the emergence of resistance as a mutation event within the P. falciparum parasite followed by natural selection and drug selection within the human host, suggests the need for modeling the interactions between the human and Plasmodium parasite population, as was the case in 56\% (5/9) of the emergence articles reviewed. The transmission of malaria occurs between the human and mosquito populations, through P. falciparum gametocytes (human-to-mosquito transmission) and P. falciparum sporozoites (mosquito-to-human transmission). The human population only was modeled in $42 \%$ $(8 / 19)$ of the transmission models, where the transmission to and from the mosquito was summarized within the equations. However, the human and mosquito populations were modeled in $32 \%(6 / 19)$ of the transmission models reviewed. When modeling the impact of antimalarial use on the emergence and transmission of resistance, the human and mosquito populations were modeled in $44 \%(4 / 9)$ articles reviewed, and the human population only in 33\% (3/9). The added complexity of modeling all three populations (human, mosquito and P. falciparum) was not common at $14 \%(5 / 37)$ across the three model categories.

As we have observed in this review, there are many factors that impact the emergence and transmission of antimalarial resistance in the human population, many of which are impacted by, or predict the effect of, drug use in the population (e.g., natural immunity, transmission intensity).

\subsection{Risk Factors for the Emergence and Transmission of Resistance}

An increase in the proportion of the population that receives antimalarial treatment (population treatment coverage) predicted an increase in the emergence and/or transmission of resistance. Risk factors contributing to the proportion of the population receiving antimalarial treatment are:

- Antimalarial treatment to resolve clinical symptoms $[6,17,18,27,35,36,38,43,50,51]$;

- Use of intermittent preventive treatment (IPT) [6];

- $\quad$ Presumptive treatment [37]; and

- $\quad$ MDAs and mass screening and treatments (MSATs) [35,36].

The exposure of parasites to residual drug concentrations (sub-optimal or sub-therapeutic API) was identified as a driver of the emergence of antimalarial resistance, and to increase the transmission of resistance. Risk factors contributing to the exposure of parasites to residual drug concentrations are:

- The presence of residual drug concentration $[8,23,29,37,45]$;

- $\quad$ Long half-lives $[6,41,43]$;

- Low antimalarial dose (API) [23];

- Low drug efficacy [22]; and 
- $\quad$ Shorter duration of high dose (API) [22].

Other treatment factors include the timing of antimalarial treatment $[19,22]$ and the use of combination therapies. The use of combination therapies was consistently predictive of a decrease in the emergence and transmission of resistance [18,24,35-37,42], provided the treatment had not previously been used as a monotherapy [24] and resistance had not emerged to one of the partner drugs [35,49]. This finding highlights the importance of the adherence to Antimalarial Treatment Guidelines proposed by the World Health Organization [53].

Other risk factors for the emergence and transmission of resistance that were included in the models reviewed can be categorized into the following groups:

- P. falciparum population: mutation rate [22,43], fitness cost of resistance $[22,25,27,30,32,44]$, degree of resistance [29,40], within-host competition [32];

- Human population: infectious period [28]; recovery rate of non-immune humans infected with resistant parasites [27]; and natural immunity [21,23] (related to transmission intensity [47]); and

- Female Anopheles mosquito population: lifespan of resistant-infected mosquitoes [27]; mosquito diffusion [27]; number of sporozoites [34]; and transmission blockers (e.g., bednets) [36,49].

\subsection{Review Limitations}

The primary limitation in the search strategy was the restriction of articles to English-language only studies. This excludes any relevant literature published in other languages. The majority of the studies reviewed were theoretical $(76 \%)$, and as such, highlighted the limitations often experienced with a lack of comprehensive datasets in this field [33].

\subsection{Implications for Further Research}

The scoping review has highlighted areas requiring further research and model development, in order to better understand the impact of antimalarial use on the emergence and transmission of resistance.

\subsubsection{Drug Selection Pressure}

Antimalarial treatments that contribute to the overall population treatment coverage are of importance. The use of IPT, MDA and MSAT strategies are areas of research that need to be evaluated further mathematically, in order to understand the impact of population treatment coverage on the emergence and/or transmission of resistance.

Antimalarial treatment that contributes to the presence of residual drug concentrations within the population is of importance. The quality, efficacy and percentage API (including high/low dose, residual API, self-medication, non-compliance) are key areas of research that need to be evaluated further mathematically, in order to understand the impact of residual concentrations on the emergence and/or transmission of resistance.

Although the effect of poor drug quality (substandard, falsified and/or degraded medicines) was not addressed in the mathematical models reviewed, it is being increasingly recognized as a source of residual drug concentrations [54]. Approximately 30\% of antimalarial medicines in Africa and Asia are considered to be substandard or falsified $[55,56]$. Substandard antimalarials are genuine medicines that generally meet the API and/or formulation content guidelines but fail to meet good manufacturing practice [54]. Degraded antimalarials leave the manufacturer as quality-assured medicines but degrade due to inadequate storage conditions (excess heat, humidity or exposure to light) [53,57]. Both substandard and degraded antimalarials can be a source of sub-therapeutic API concentrations. Falsified medicines are fraudulently mislabeled with respect to identity and/or source $[54,58,59]$, the quality and manufacture of which are not regulated by government health sectors to ensure internationally recognized standards are met. The API may be correct or incorrect 
with regards to ingredient and dose, or they may contain incorrect ingredients [60]. As a result of poor formulation techniques, falsified medicines may release an incorrect amount of API and expose parasites to sub-therapeutic API concentrations. The impact of the use of poor-quality antimalarial medicines on the emergence and transmission of resistance needs to be explored as they may be a significant contributor to the drivers of resistance identified in this review: population treatment coverage and residual concentrations.

The practice of self-diagnosis (self-medication) was also not addressed in the mathematical models reviewed. This practice can result in the patient having the wrong treatment regimen, a shorter treatment period than is recommended, and/or an incorrect dose of API(s) (poor quality antimalarials, or quality-assured antimalarials that have expired or degraded). Self-medication is commonly identified to be a contributor to the population treatment coverage, without a malaria diagnosis [4]. As the API declines with time, this provides a selective pressure on any future infections, adding to the residual concentrations within the population. These areas of future research are outlined in Table 2.

Table 2. Areas of future mathematical modeling research that contribute to the main drivers of the emergence and transmission of resistance identified in the scoping review, where gaps are indicated by ' $X$ '.

\begin{tabular}{|c|c|c|c|}
\hline Treatment Scenario & Emergence & Transmission & Emergence and Transmission \\
\hline \multicolumn{4}{|c|}{ Contributing to population treatment coverage } \\
\hline IPT use & $x$ & & \\
\hline MDA & $X$ & & \\
\hline MSAT & $x$ & & $x$ \\
\hline Self-medication & $x$ & $x$ & $x$ \\
\hline \multicolumn{4}{|c|}{ Contributing to residual drug concentrations } \\
\hline Drug efficacy & & $x$ & $x$ \\
\hline $\begin{array}{c}\text { Drug quality (falsified, substandard and } \\
\text { degraded) }\end{array}$ & $x$ & $x$ & $\mathrm{X}$ \\
\hline $\begin{array}{l}\text { Full/partial treatment and patient } \\
\text { compliance }\end{array}$ & $x$ & & $x$ \\
\hline High/low dose & & $x$ & \\
\hline Percentage API & $x$ & $x$ & $x$ \\
\hline Residual/subtherapeutic API & & & $x$ \\
\hline Self-medication & $x$ & $x$ & $x$ \\
\hline
\end{tabular}

The lifecycle of the P. falciparum parasite has stages within the human host and the female Anopheles vector. In order to fully understand the impact of antimalarial use on the emergence and transmission of $P$. falciparum drug resistance in humans, we propose that a review of the effect of drug use on Plasmodium spp. parasite populations needs to be carried out. The combination of this scoping review with the proposed review would provide a deeper understanding of the underlying dynamics.

\subsubsection{Influencing Factors}

The infectivity of gametocytes following treatment, taking into account the antimalarial treatment and quality, must be explored in order to fully understand how the transmission of resistance is affected by antimalarial use.

Conflicting findings of the impact of natural immunity, transmission intensity, and transmission blockers on the emergence and transmission of resistance were revealed in this review, and provide areas for further research.

\section{Conclusions}

The emergence and transmission of resistance to antimalarial treatments are continuing to hamper malaria elimination efforts. This article has reviewed the use of mathematical models to describe the relationship between antimalarial use and P. falciparum antimalarial resistance within the human population, and identified other factors that influence this relationship. However, this scoping review 
has identified key areas for future research in order to better understand the impact of antimalarial use on the emergence and transmission of antimalarial resistance.

Supplementary Materials: The following are available online at http://www.mdpi.com/2414-6366/2/4/54/s1, Table S1: Medline and Embase Database Search, Table S2: CINAHL Database Search, Table S3: Web of Science Database Search, Table S4: Cochrane Library Database Search, Table S5: Scopus Database Search, and Table S6: Grey Literature Search (Google Scholar and Open Grey).

Acknowledgments: A.R.B. is supported by a University of South Australia Postgraduate Award.

Author Contributions: A.R.B. and A.E. conceived the study; A.R.B. carried out the database searches under the guidance of C.A.G.; A.R.B. carried out the screening and selection of articles against the criteria, with validation provided by A.E. and J.V.R.; A.R.B. analyzed the articles, with assistance from J.V.R.; A.R.B. wrote the draft manuscript, with feedback provided by J.V.R., A.E. and C.A.G.

Conflicts of Interest: The authors declare no conflict of interest.

\section{References}

1. WHO. World Malaria Report 2016; World Health Organization: Geneva, Switzerland, 2016.

2. White, N.J.; Pongtavornpinyo, W. The de novo selection of drug-resistant malaria parasites. Proc. Biol. Sci. 2003, 270, 545-554. [CrossRef] [PubMed]

3. Valderramos, S.; Valderramos, J.; Musset, L.; Purcell, L.; Mercereau-Puijalon, O.; Legrand, E.; Fidock, D.A. Identification of a mutant PfCRT-mediated chloroquine tolerance phenotype in Plasmodium falciparum. PLoS Pathog. 2010, 6, e1000887. [CrossRef] [PubMed]

4. WHO. Global Report on Antimalarial Drug Efficacy and Drug Resistance: 2000-2010; Drug Resistance and Containment Unit, Global Malaria Programme, World Health Organization: Geneva, Switzerland, 2010.

5. White, N.J. Antimalarial drug resistance. J. Clin. Investig. 2004, 113, 1084-1092. [CrossRef] [PubMed]

6. O'Meara, W.P.; Smith, D.L.; McKenzie, F.E. Potential impact of intermittent preventive treatment (IPT) on spread of drug-resistant malaria. PLoS Med. 2006, 3, e141.

7. Hastings, I.M.; Paget-McNicol, S.; Saul, A. Can mutation and selection explain virulence in human P. falciparum infections? Malar. J. 2004, 3, 2. [CrossRef] [PubMed]

8. Pongtavornpinyo, W.; Hastings, I.M.; Dondorp, A.; White, L.J.; Saralamba, S.; Day, N.P.; White, N.J.; Boni, M.F. Probability of emergence of antimalarial resistance in different stages of the parasite life cycle. Evol. Appl. 2009, 2, 52-61. [CrossRef] [PubMed]

9. Walliker, D. Malaria. In Molecular Epidemiology of Infectious Diseases; Thompson, R.A., Ed.; Arnold: London, UK, 2000; pp. 93-112.

10. Walliker, D.; Hunt, P.; Babiker, H. Fitness of drug-resistant malaria parasites. Acta. Trop. 2005, 94, 251-259. [CrossRef] [PubMed]

11. Mandal, S.; Sarkar, R.R.; Sinha, S. Mathematical models of malaria-A review. Malar. J. 2011, 10, 202. [CrossRef] [PubMed]

12. White, L.J.; Maude, R.J.; Pongtavornpinyo, W.; Saralamba, S.; Aguas, R.; Van Effelterre, T.; Day, N.P.J.; White, N.J. The role of simple mathematical models in malaria elimination strategy design. Malar. J. 2009, 8, 212. [CrossRef] [PubMed]

13. Arksey, H.; O'Malley, L. Scoping studies: Towards a methodological framework. Int. J. Soc. Res. Methodol. 2005, 8, 19-32. [CrossRef]

14. Levac, D.; Colquhoun, H.; O’Brien, K.K. Scoping studies: Advancing the methodology. Implement. Sci. 2010, 5, 69. [CrossRef] [PubMed]

15. Colquhoun, H.L.; Levac, D.; O’Brien, K.K.; Straus, S.; Tricco, A.C.; Perrier, L.; Kastner, M.; Moher, D. Scoping reviews: Time for clarity in definition, methods, and reporting. J. Clin. Epidemiol. 2014, 67, e1291-e1294. [CrossRef] [PubMed]

16. Covidence Systematic Review Software. Available online: www.covidence.org (accessed on 9 October 2017).

17. Cross, A.P.; Singer, B. Modelling the development and resistance of Plasmodium falciparum to anti-malarial drugs. Trans. R. Soc. Trop. Med. Hyg. 1991, 85, 349-355. [CrossRef]

18. Curtis, C.F.; Otoo, L.N. A simple model of the build-up of resistance to mixtures of anti-malarial drugs. Trans. R. Soc. Trop. Med. Hyg. 1986, 80, 889-892. [CrossRef] 
19. Gatton, M.L.; Hogarth, W.; Saul, A. Time of treatment influences the appearance of drug-resistant parasites in Plasmodium falciparum infections. Parasitology 2001, 123, 537-546. [CrossRef] [PubMed]

20. Hastings, I.M. A model for the origins and spread of drug-resistant malaria. Parasitology 1997, 115, $133-141$. [CrossRef] [PubMed]

21. Janssen, M.A.; Martens, W.J.M. Modeling malaria as a complex adaptive system. Artif. Life 1997, 3, $213-236$. [CrossRef] [PubMed]

22. Kim, Y.; Escalante, A.A.; Schneider, K.A. A population genetic model for the initial spread of partially resistant malaria parasites under anti-malarial combination therapy and weak intrahost competition. PLOS ONE 2014, 9, e101601. [CrossRef] [PubMed]

23. Simpson, J.A.; Watkins, E.R.; Price, R.N.; Aarons, L.; Kyle, D.E.; White, N.J. Mefloquine pharmacokinetic-pharmacodynamic models: Implications for dosing and resistance. Antimicrob. Agents Chemother. 2000, 44, 3414-3424. [CrossRef] [PubMed]

24. Watkins, W.M.; Sibley, C.H.; Hastings, I.M. The search for effective and sustainable treatments for Plasmodium falciparum malaria in Africa: A model of the selection of resistance by antifolate drugs and their combinations. Am. J. Trop. Med. Hyg. 2005, 72, 163-173. [PubMed]

25. Antao, T.; Hastings, I.M. Environmental, pharmacological and genetic influences on the spread of drug-resistant malaria. Proc. R. Soc. B 2011, 278, 1705-1712. [CrossRef] [PubMed]

26. Artzy-Randrup, Y.; Alonso, D.; Pascual, M. Transmission intensity and drug resistance in malaria population dynamics: Implications for climate change. PLOS ONE 2010, 5, e13588. [CrossRef] [PubMed]

27. Bacaer, N.; Sokhna, C. A reaction-diffusion system modeling the spread of resistance to an antimalarial drug. Math. Biosci. Eng. 2005, 2, 227-238. [PubMed]

28. Chiyaka, C.; Garira, W.; Dube, S. Effects of treatment and drug resistance on the transmission dynamics of malaria in endemic areas. Theor. Popul. Biol. 2009, 75, 14-29. [CrossRef] [PubMed]

29. Gatton, M.L.; Cheng, Q. Plasmodium falciparum infection dynamics and transmission potential following treatment with sulfadoxine-pyrimethamine. J. Antimicrob. Chemother. 2006, 58, 47-51. [CrossRef] [PubMed]

30. Hastings, I.M. Complex dynamics and stability of resistance to antimalarial drugs. Parasitology 2006, 132, 615-624. [CrossRef] [PubMed]

31. Klein, E.Y.; Smith, D.L.; Boni, M.F.; Laxminarayan, R. Clinically immune hosts as a refuge for drug-sensitive malaria parasites. Malar. J. 2008, 7, 67. [CrossRef] [PubMed]

32. Klein, E.Y.; Smith, D.L.; Laxminarayan, R.; Levin, S. Superinfection and the evolution of resistance to antimalarial drugs. Proc. R. Soc. B 2012, 279, 3834-3842. [CrossRef] [PubMed]

33. Koella, J.; Antia, R. Epidemiological models for the spread of anti-malarial resistance. Malar J. $2003,2,3$. [CrossRef] [PubMed]

34. Mackinnon, M.J.; Hastings, I.M. The evolution of multiple drug resistance in malaria parasites. Trans. R. Soc. Trop. Med. Hyg. 1998, 92, 188-195. [CrossRef]

35. Maude, R.J.; Socheat, D.; Nguon, C.; Saroth, P.; Dara, P.; Li, G.; Song, J.; Yeung, S.; Dondorp, A.M.; Day, N.P.; et al. Optimising strategies for Plasmodium falciparum malaria elimination in Cambodia: Primaquine, mass drug administration and artemisinin resistance. PLoS ONE 2012, 7, e37166. [CrossRef] [PubMed]

36. Maude, R.J.; Pontavornpinyo, W.; Saralamba, S.; Aguas, R.; Yeung, S.; Dondorp, A.M.; Day, N,P.; White, N.J.; White, L.J. The last man standing is the most resistant: Eliminating artemisinin-resistant malaria in Cambodia. Malar. J. 2009, 8, 31.

37. Pongtavornpinyo, W.; Yeung, S.; Hastings, I.M.; Dondorp, A.M.; Day, N.P.J.; White, N.J. Spread of anti-malarial drug resistance: Mathematical model with implications for ACT drug policies. Malar. J. 2008, 7, 299. [CrossRef] [PubMed]

38. Schneider, K.A.; Kim, Y. An analytical model for genetic hitchhiking in the evolution of antimalarial drug resistance. Theor. Popul. Biol. 2010, 78, 93-108. [CrossRef] [PubMed]

39. Slater, H.C.; Griffin, J.T.; Ghani, A.C.; Okell, L.C. Assessing the potential impact of artemisinin and partner drug resistance in sub-Saharan Africa. Malar. J. 2016, 15, 10. [CrossRef] [PubMed]

40. Tasman, H.; Soewono, E.; Sidarto, K.A. A model for transmission of partial resistance to anti-malarial drugs. Math. Biosci. Eng. 2009, 6, 649-661. [CrossRef] [PubMed] 
41. Teboh-Ewungkem, M.I.; Mohammed-Awel, J.; Baliraine, F.N.; Duke-Sylvester, S.M. The effect of intermittent preventive treatment on anti-malarial drug resistance spread in areas with population movement. Malar. J. 2014, 13, 428. [CrossRef] [PubMed]

42. Winter, K.; Hastings, I.M. Development, evaluation, and application of an in silico model for antimalarial drug treatment and failure. Antimicrob. Agents Chemother. 2011, 55, 3380-3392. [CrossRef] [PubMed]

43. An, L.T.T.; Jäger, W. A quantitative model of population dynamics in malaria with drug treatment. J. Math. Biol. 2014, 69, 659-685. [CrossRef] [PubMed]

44. Esteva, L.; Gumel, A.B.; de León, C.V. Qualitative study of transmission dynamics of drug-resistant malaria. Math. Comput. Model. 2009, 50, 611-630. [CrossRef]

45. Hastings, I.M.; Watkins, W.M.; White, N.J. The evolution of drug-resistant malaria: The role of drug elimination half-life. Philoso. Trans. R. Soc. B 2002, 357, 505-519. [CrossRef] [PubMed]

46. Klein, E.Y. The impact of heterogeneous transmission on the establishment and spread of antimalarial drug resistance. J. Theor. Biol. 2014, 340, 177-185. [CrossRef] [PubMed]

47. Legros, M.; Bonhoeffer, S. A combined within-host and between-hosts modelling framework for the evolution of resistance to antimalarial drugs. J. R. Soc. Interface 2016, 13. [CrossRef] [PubMed]

48. Mackinnon, M.J. Drug resistance models for malaria. Acta. Trop. 2005, 94, 207-217. [CrossRef] [PubMed]

49. Maude, R.J.; Nguon, C.; Dondorp, A.M.; White, L.J.; White, N.J. The diminishing returns of atovaquone-proguanil for elimination of Plasmodium falciparum malaria: Modelling mass drug administration and treatment. Malar. J. 2014, 13, 380. [CrossRef] [PubMed]

50. Smith, D.L.; Klein, E.Y.; McKenzie, F.E.; Laxminarayan, R. Prospective strategies to delay the evolution of anti-malarial drug resistance: Weighing the uncertainty. Malar. J. 2010, 9, 217. [CrossRef] [PubMed]

51. Tchuenche, J.M.; Chiyaka, C.; Chan, C.; Matthews, A.; Mayer, G. A mathematical model for antimalarial drug resistance. Math. Med. Biol. 2011, 28, 335-355. [CrossRef] [PubMed]

52. Bloland, P.B.; Williams, H.A.; National Research Council; Committee on Population. Malaria Control during Mass Population Movements and Natural Disasters; The National Academies Press: Washington, DC, USA, 2003.

53. WHO. Guidelines for the Treatment of Malaria, 3rd edition; World Health Organization: Geneva, Switzerland, 2015.

54. Bassat, Q.; Tanner, M.; Guerin, P.J.; Stricker, K.; Hamed, K. Combating poor quality antimalarial medicines: A call to action. Malar. J. 2016, 15, 302. [CrossRef] [PubMed]

55. Ambroise-Thomas, P. The tragedy caused by fake antimalarial drugs. Mediterr. J. Hematol. Infect. Dis. 2012, 4, e2012027. [CrossRef] [PubMed]

56. Newton, P.; Green, M.; Fernandez, F. Impact of poor quality-quality medicines in the 'developing' world. Trends Pharmacol. Sci. 2009, 31, 99-101. [CrossRef] [PubMed]

57. Newton, P.N.; Caillet, C.; Guerin, P.J. A link between poor quality antimalarials and malaria drug resistance? Expert Rev. Anti Infect. Ther. 2016, 14, 531-533. [CrossRef] [PubMed]

58. Newton, P.N.; Green, M.D.; Fernandez, F.; Day, N.P.; White, N.J. Counterfeit anti-infective drugs. Lancet Infect. Dis. 2006, 6, 602-613. [CrossRef]

59. Harper, J.; Morris, J.; Satchwell, G.; Stevens, P.; Taylor, D.; Tremblay, M. Coincidence or Crisis? Prescription Medicine Counterfeiting; Pitts, P.J., Ed.; The Stockholm Network: London, UK, 2006.

60. Newton, P.N.; Fernandez, F.M.; Plancon, A.; Mildenhall, D.C.; Green, M.D.; Ziyong, L.; Christophel, E.M.; Phanouvong, S.; Howells, S.; McIntosh, E.; et al. A collaborative epidemiological investigation into the criminal fake artesunate trade in South East Asia. PLoS Med. 2008, 5, e32. [CrossRef] [PubMed]

(C) 2017 by the authors. Licensee MDPI, Basel, Switzerland. This article is an open access article distributed under the terms and conditions of the Creative Commons Attribution (CC BY) license (http://creativecommons.org/licenses/by/4.0/). 